\title{
Time Course of Ventricular Remodeling after Atrial Septal Defect Closure in Adult Patients
}

\author{
Yo Han Bae, M.D. ', Woo Sung Jang, M.D., Ph.D.', Jin Young Kim, M.D. ${ }^{2}$, Yun Seok Kim, M.D., Ph.D. ${ }^{1}$ \\ Departments of 'Thoracic and Cardiovascular Surgery and ${ }^{2}$ Radiology, Keimyung University Dongsan Hospital, Keimyung University College of Medicine, Daegu, \\ Korea
}

\section{ARTICLE INFO}

Received July 16, 2020

Revised September 9, 2020

Accepted October 5, 2020

Corresponding author

Woo Sung Jang

Tel 82-52-258-7966

Fax 82-53-258-4783

E-mail whiteuri@dsmc.or.kr

ORCID

https://orcid.org/0000-0003-1576-9472
Background: Atrial septal defect (ASD) is the most common congenital heart disease. However, the details of cardiac chamber remodeling after surgery are not well known, although this is an important issue that should be analyzed to understand long-term outcomes.

Methods: Between November 2017 and January 2019, cardiac magnetic resonance imaging was performed preoperatively, at a 1-month postoperative follow-up, and at a 1-year postoperative follow-up. Cardiac chamber volume, valve regurgitation volume, and ejection fraction were measured as functions of time.

Results: Thirteen patients (10 men and 3 women) were included. The median age at surgery was 51.4 years. The preoperative median ratio of flow in the pulmonary and systemic circulation was 2.3. The preoperative mean right ventricular (RV) end-diastolic volume index (EDVi) and RV end-systolic volume index (ESVi) had significantly decreased at the 1-month postoperative follow-up ( $p<0.001, p=0.001$, respectively). The decrease in the RVEDVi $(p=0.085)$ and RVESVi $(p=0.023)$ continued until the postoperative 1-year follow-up, although the rate of decrease was slower. Tricuspid valve regurgitation had also decreased at the 1-month postoperative follow-up ( $p=0.022)$, and continued to decrease at a reduced rate $(p=0.129)$. Although the RVEDVi and RVESVi improved after ASD closure, the RV volume parameters were still larger than the left ventricular (LV) volume parameters at the 1-year follow-up (RVEDVi vs. LVEDVi: $p=0.016$; RVESVi vs. LVESVi: $p=0.001$ ).

Conclusion: Cardiac remodeling after ASD closure is common and mainly occurs in the early postoperative period. However, complete normalization does not occur.

Keywords: Atrial heart septal defects, Cardiac magnetic resonance imaging, Congenital heart disease

\section{Introduction}

Atrial septal defect (ASD) is the most common congenital heart disease, accounting for approximately $10 \%-15 \%$ of all cases of congenital heart disease [1]. Quantifying the ratio of flow in the pulmonary $(\mathrm{Qp})$ and systemic circulation (Qs) is essential for detecting the amount shunted from the left to right side of the atrium, and a Qp/Qs over 1.5 indicates enlargement of the right ventricle (RV) and predicts symptoms including exercise intolerance, fatigue, dyspnea, and palpitation [2]. Thus, interventional closure or corrective surgical surgery should be considered to treat ASD.
Long-term outcomes after ASD closure are favorable, although there remains a risk of arrhythmia with concomitant neurological events after ASD closure [3]. For this reason, cardiac chamber remodeling after surgery is an important issue after ASD closure that should be analyzed to understand the long-term outcomes. However, the extent and time course of cardiac remodeling after ASD closure have not been thoroughly characterized. Thus, the aim of this study was to evaluate the time course of ventricular remodeling by cardiac magnetic resonance imaging (CMR). 


\section{Methods}

\section{Study population}

This study protocol was approved by the Institutional Review Board of Keimyung University Dongsan Hospital (IRB approval no., 2017-10-024), and all procedures were performed in accordance with our institutional guidelines for the protection of patient confidentiality.

Between November 2017 and January 2019, among the patients who underwent surgical ASD closure, 13 patients (10 women and 3 men) who had consented to the CMR protocol were included. Informed consent was obtained from all patients.

\section{Data collection}

Data on demographics, clinical and laboratory findings, and treatment outcomes were obtained with data collection forms from patients' electronic medical records. The demographic data included age, sex, body weight, predefined comorbidities (hypertension, diabetes, coronary artery disease, chronic obstructive pulmonary disease, and chronic kidney disease), and symptoms (chest discomfort, dyspnea, and palpitation). Laboratory data included N-terminal probrain natriuretic peptide (NT proBNP).

The CMR data included the right ventricular end-diastolic volume index (RVEDVi), right ventricular end-systolic index (RVESVi), right ventricular ejection fraction (RVEF), tricuspid valve regurgitation (TVR), left ventricular end-diastolic volume index (LVEDVi), left ventricular end-systolic index (LVESVi), left ventricular ejection fraction (LVEF), and mitral valve regurgitation (MVR). In addition, late gadolinium enhancement (LGE) images were also obtained. CMR was performed 3 times: preoperatively and at 1-month and 1-year postoperative follow-up visits. The NT proBNP level was also checked at the same time as CMR.

\section{Cardiac magnetic resonance imaging protocol}

All subjects underwent CMR with a 3T scanner (Ingenia CX; Philips Medical Systems, Best, the Netherlands). An 18-element body matrix coil and a spine matrix coil array were used. Cine images were acquired in the short-axis plane orientation by using a retrospectively echocardiography-gated balanced steady-state free precession sequence with the following parameters: repetition time (TR)/echo time (TE), 3/1.52 ms; flip angle (FA), $45^{\circ}$; slice thickness
(ST), $8 \mathrm{~mm}$; slice gap, $8 \mathrm{~mm}$; matrix, $172 \times 159$ pixels; and field of view (FOV), $340 \times 340 \mathrm{~mm}^{2}$. Gadolinium contrast agent (Uniary; Dongkook Life Science, Seoul, Korea) was intravenously administered at $0.2 \mathrm{mmol} / \mathrm{kg}$. LGE CMR images were acquired 15 minutes after contrast injection by using a magnitude- and phase-sensitive inversion-recovery-prepared sequence with inversion time adjusted to null, thus representing the normal myocardium. These LGE images were obtained along the same axis plane covering the whole left ventricle (LV) (TR, $6.1 \mathrm{~ms}$; TE, $3 \mathrm{~ms}$; FOV, $350 \times 350 \mathrm{~mm}^{2}$; ST, $8 \mathrm{~mm}$; acquisition matrix, $220 \times 161$ pixels; nonselective inversion recovery FA, $25^{\circ}$; and bandwidth, 236.7 Hz/pixel). The appropriate inversion time prior to LGE MR was determined using a fast low-angle shot sequence with varying inversion times (from 150 to $650 \mathrm{~ms}$ to null).

\section{Cardiac magnetic resonance imaging image analysis}

Ventricular end-diastolic and end-systolic volumes were obtained by delineating the endocardial borders of the LV and RV in all slices of the short-axis stack. All ventricular volumes were indexed to body surface area. We obtained

Table 1. Preoperative patient characteristics

\begin{tabular}{|c|c|c|}
\hline Characteristic & Preoperative & $\begin{array}{c}\text { Postoperative } \\
\text { 1-year follow-up }\end{array}$ \\
\hline Age (yr) & $51.4(41.4-58.4)$ & \\
\hline \multicolumn{3}{|l|}{ Sex } \\
\hline Male & $3(23.1)$ & \\
\hline Female & $10(76.9)$ & \\
\hline Smoking & $1(7.7)$ & \\
\hline \multicolumn{3}{|l|}{ Comorbidities } \\
\hline Hypertension & $2(15.4)$ & \\
\hline Diabetes mellitus & $1(7.7)$ & \\
\hline Coronary artery disease & 0 & \\
\hline $\begin{array}{l}\text { Chronic obstructive } \\
\text { pulmonary disease }\end{array}$ & 0 & \\
\hline Chronic kidney disease & 0 & \\
\hline \multicolumn{3}{|l|}{ Symptoms } \\
\hline Chest discomfort & $5(38.5)$ & $1(7.7)$ \\
\hline Dyspnea & $4(30.8)$ & $4(30.8)$ \\
\hline \multirow[t]{4}{*}{ NYHA FC } & III & II \\
\hline & II & II \\
\hline & II & I \\
\hline & II & I \\
\hline Palpitation & $2(15.4)$ & $2(15.4)$ \\
\hline
\end{tabular}

Values are presented as median (interquartile range) or number (\%), unless otherwise stated.

NYHA FC, New York Heart Association functional class. 
both the ventricular ejection fraction and the shunt ratio between pulmonary and aortic flow (Qp/Qs). The shunt volume per heartbeat was calculated from the difference in flow per heartbeat between the pulmonary trunk and aorta. We measured the TVR and MVR volumes.

\section{Surgical techniques}

All surgical procedures were performed using a standard sternotomy or right thoracotomy approach. In patients with tricuspid annular dilatation greater than $40 \mathrm{~mm}$ based on preoperative echocardiography, we performed tricuspid valve annuloplasty.

\section{Statistical analysis}

All continuous variables are expressed using median values with interquartile ranges (IQRs), as appropriate. We made no assumptions about missing data. Categorical variables are expressed as frequencies and percentages. The paired t-test was used to compare differences in CMR parameters across different time intervals. A p-value of less than 0.05 was considered to indicate statistical significance. All analyses were performed using IBM SPSS ver. 26.0 (IBM Corp., Armonk, NY, USA).
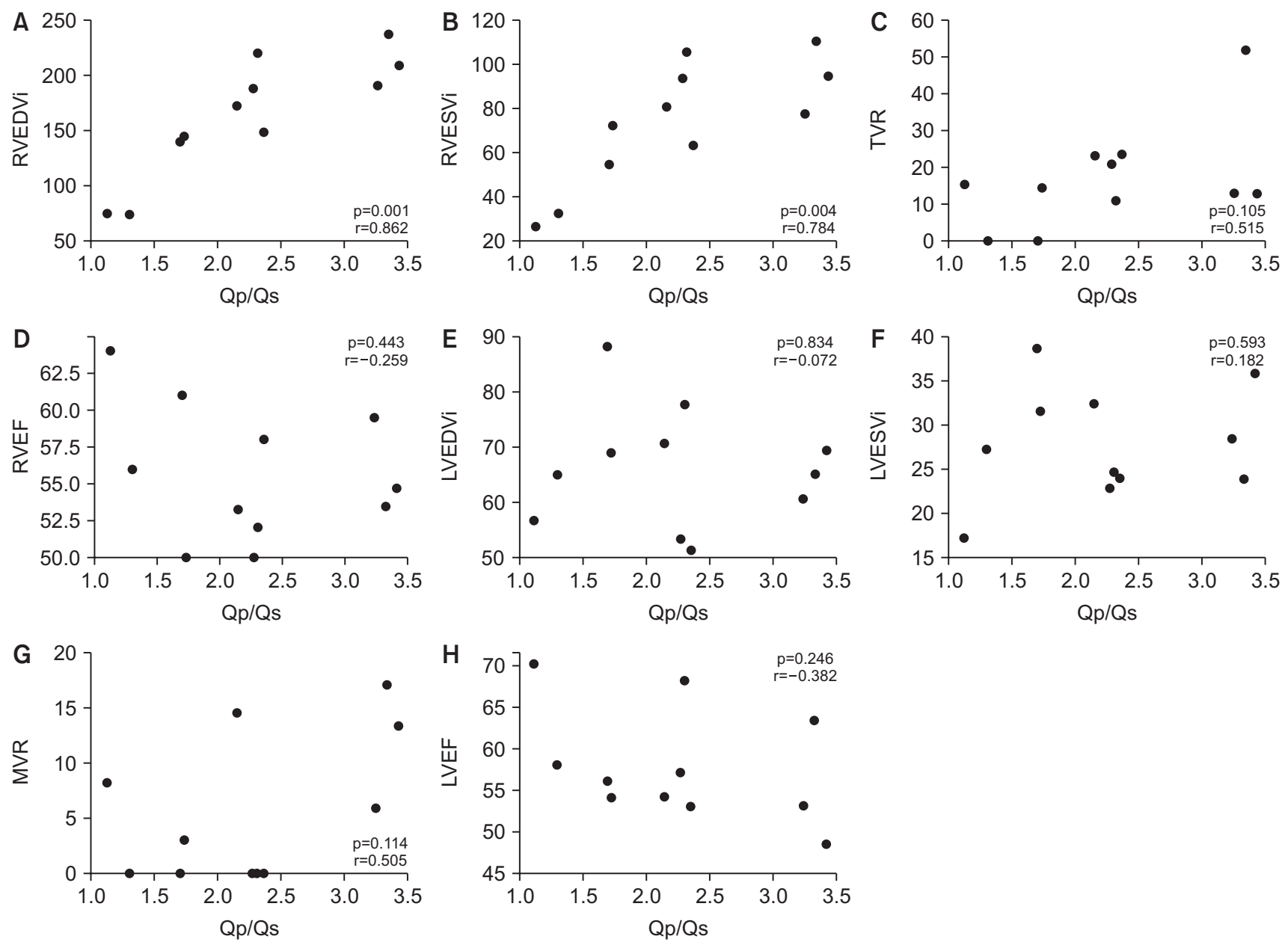

Fig. 1. Preoperative relationships between $\mathrm{Qp} / \mathrm{Qs}$ and other cardiac parameters obtained by cardiac magnetic resonance imaging. There were positive correlations between $\mathrm{Qp} / \mathrm{Qs}$ and the right ventricular size parameters. $\mathrm{Qp} / \mathrm{Qs}$, the ratio of flow in the pulmonary and systemic circulation; RVEDVi, right ventricular end-diastolic volume index; RVESVi, right ventricular end-systolic volume index; TVR, tricuspid valve regurgitation; RVEF, right ventricular ejection fraction; LVEDVi, left ventricular end-diastolic volume index; LVESVi, left ventricular end-systolic volume index; MVR, mitral valve regurgitation; LVEF, left ventricular ejection fraction. 


\section{Results}

\section{Clinical outcomes}

The patients' median age and body weight at the time of surgery were 51.4 years (IQR, 41.4-58.4 years) and $62.2 \mathrm{~kg}$ (IQR, 52-71.3 kg), respectively. As preoperative comorbidities, 2 patients (15.4\%) had hypertension and 1 patient (7.7\%) had diabetes. Their preoperative symptoms were chest discomfort in 5 patients (38.5\%), dyspnea in 4 patients (30.8\%), and palpitation in 2 patients (15.4\%). No patients had atrial fibrillation on preoperative electrocardiography.

We performed ASD patch closure in 12 patients $(92.3 \%)$ and ASD primary closure in 1 patient (7.7\%). Associated procedures included tricuspid valve annuloplasty in 1 patient $(7.7 \%)$. There were no early or late deaths related to the operations. There was no surgery-related morbidity. At the 1-year follow-up, chest discomfort and dyspnea at the preoperative period had improved in 4 patients $(80 \%, 4$ of 5 patients) and 3 patients (75\%, 3 of 4 patients), respectively. However, the 2 patients with palpitations in this study did not show symptom improvement at follow-up (Table 1).

The preoperative median NT proBNP level $(83.0 \mathrm{pg} / \mathrm{mL}$; IQR, 46.0-162.0 pg/mL) showed an increasing tendency at the 1-month postoperative follow-up $(167.0 \mathrm{pg} / \mathrm{mL}$; IQR, 113.0-342.5 pg/mL; $\mathrm{p}=0.059)$. However, the NT proBNP level then showed a decreasing tendency based on the levels at the 1-year postoperative follow-up (105.5 pg/mL; IQR, 75.3-154.5 pg/mL; $\mathrm{p}=0.118$ ).
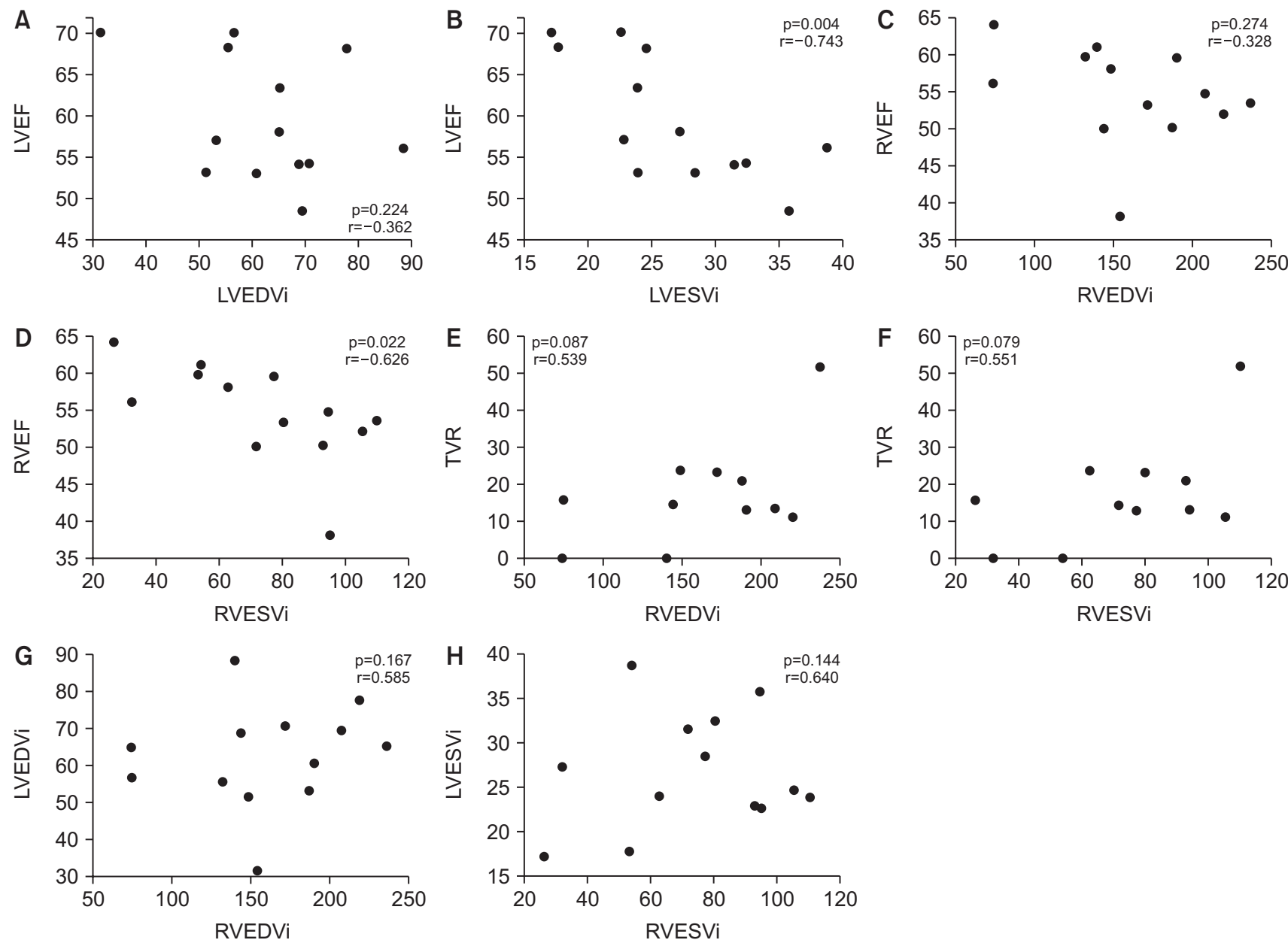

Fig. 2. Relationships between cardiac magnetic resonance imaging parameters. The ejection fraction showed a negative correlation with the ventricular end systolic volume index. LVEF, left ventricular ejection fraction; LVEDVi, left ventricular end-diastolic volume index; LVESVi, left ventricular end-systolic volume index; RVEF, right ventricular ejection fraction; RVEDVi, right ventricular end-diastolic volume index; RVESVi, right ventricular end-systolic volume index; TVR, tricuspid valve regurgitation. 


\section{Correlations of cardiac magnetic resonance imaging parameters}

The preoperative median Qp/Qs was 2.3 (IQR, 1.7-2.8). There was a positive correlation between RV size parameters and preoperative Qp/Qs (RVEDVi: $\mathrm{r}=0.862, \mathrm{p}=0.001$; RVESVi: $r=0.784, p=0.004)$. However, there were no statistically significant correlations between LV size parameters and the preoperative Qp/Qs (LVEDVi: $\mathrm{r}=-0.072, \mathrm{p}=0.834$; LVESVi: $r=0.593, p=0.593$ ) (Fig. 1).

The RVEF and LVEF were negatively correlated with RVESVi $(\mathrm{r}=-0.626, \mathrm{p}=0.022)$ and LVESVi $(\mathrm{r}=-0.743$, $\mathrm{p}=0.004)$, respectively. However, there was no meaningful correlation between the RVEF and LVEF and RVEDVi and
LVEDVi, respectively (RVEDVi-RVEF: $\mathrm{r}=-0.328, \mathrm{p}=0.274$; LVEDVi-LVEF: $\mathrm{r}=-0.362, \mathrm{p}=0.224)$. Although there was no significant difference, the amount of TVR was correlated with RVEDVi ( $\mathrm{r}=0.539, \mathrm{p}=0.087)$ and RVESVi ( $\mathrm{r}=0.551$, $\mathrm{p}=0.079$ ) (Fig. 2). There were no significant correlations between NT proBNP levels and any of the CMR parameters $(\mathrm{p}>0.05$ for all).

\section{Time course of ventricular remodeling after atrial septal defect closure}

\section{Cardiac magnetic resonance imaging parameters}

The preoperative mean RVEDVi and RVESVi had significantly decreased at the postoperative follow-up at 1
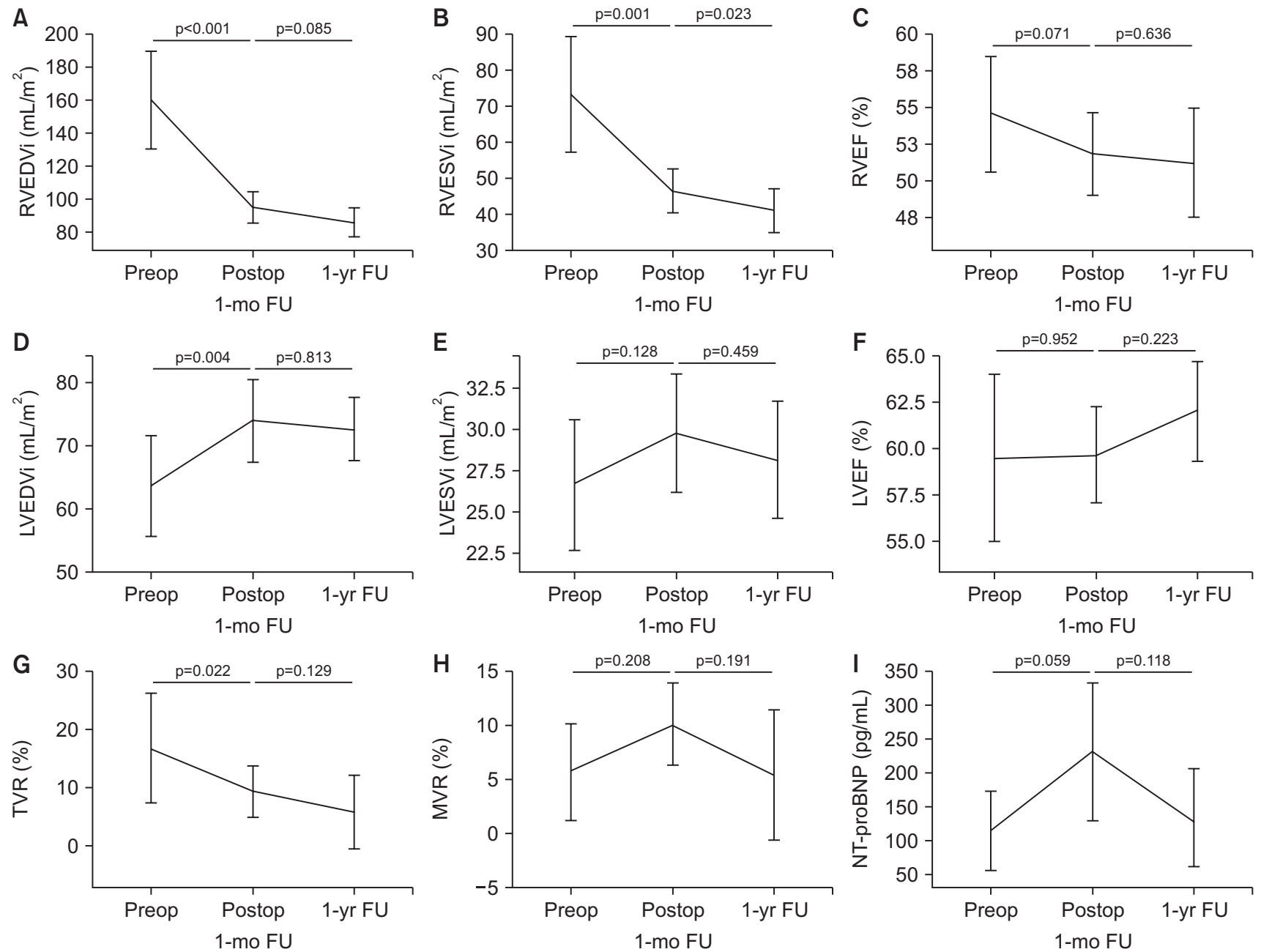

Fig. 3. Ventricular remodeling during FU on cardiac magnetic resonance imaging. Preop, preoperative; Postop, postoperative; FU, follow-up; RVEDVi, right ventricular end-diastolic volume index; RVESVi, right ventricular end-systolic volume index; RVEF, right ventricular ejection fraction; LVEDVi, left ventricular end-diastolic volume index; LVESVi, left ventricular end-systolic volume index; LVEF, left ventricular ejection fraction; TVR, tricuspid valve regurgitation; MVR, mitral valve regurgitation; NT-proBNP, N-terminal pro-brain natriuretic peptide. 
Table 2. Cardiac magnetic resonance imaging parameters during FU

\begin{tabular}{lccc}
\hline \multicolumn{1}{c}{ Variable } & Preoperative & Postoperative FU at 1 month & Postoperative FU at 1 year \\
\hline RVEDVi $\left(\mathrm{mL} / \mathrm{m}^{2}\right)$ & $154.0(139.6-190.3)$ & $97.1(84.9-103.9)$ & $81.5(72.3-97.2)$ \\
RVESVi $\left(\mathrm{mL} / \mathrm{m}^{2}\right)$ & $77.1(54.1-94.3)$ & $48.1(42.9-50.3)$ & $39.5(36.1-45.6)$ \\
RVEF $(\%)$ & $54.7(52.0-59.5)$ & $51.6(51.0-55.0)$ & $51.0(49.9-54.7)$ \\
LVEDVi $\left(\mathrm{mL} / \mathrm{m}^{2}\right)$ & $64.8(55.3-69.2)$ & $72.5(67.0-79.2)$ & $68.8(66.7-74.2)$ \\
LVESVi $\left(\mathrm{mL} / \mathrm{m}^{2}\right)$ & $24.6(22.8-31.5)$ & $29.3(26.8-33.7)$ & $27.9(23.7-29.8)$ \\
LVEF $(\%)$ & $57.0(54.0-68.0)$ & $60.2(57.5-63.0)$ & $63.0(58.8-65.2)$ \\
TVR $(\%)$ & $14.1(11.7-21.8)$ & $8.3(4.5-14.4)$ & $0(0-8.9)$ \\
MVR $(\%)$ & $3.0(0-10.7)$ & $11.3(6.9-13.7)$ & $0(0-9.32)$ \\
\hline
\end{tabular}

Values are presented as median (interquartile range).

FU, follow-up; RVEDVi, right ventricular end-diastolic volume index; RVESVi, right ventricular end-systolic volume index; RVEF, right ventricular ejection fraction; LVEDVi, left ventricular end-diastolic volume index; LVESVi, left ventricular end-systolic volume index; LVEF, left ventricular ejection fraction; TVR, tricuspid valve regurgitation; MVR, mitral valve regurgitation.

month ( $\mathrm{p}<0.001, \mathrm{p}=0.001$, respectively). The RVEDVi $(\mathrm{p}=0.085)$ and RVESVi $(\mathrm{p}=0.023)$ continued to decrease until the postoperative follow-up at 1 year, although the rate of decrease slowed. TVR had also decreased at the 1-month postoperative follow-up $(\mathrm{p}=0.022)$, and the TVR decrease continued, albeit more slowly $(\mathrm{p}=0.129)$. The preoperative mean LVEDVi and LVESVi had increased at the 1 -month postoperative follow-up ( $\mathrm{p}=0.004$ and $\mathrm{p}=0.128$, respectively) and then remained unchanged at the 1-year postoperative follow-up ( $\mathrm{p}=0.813$ and $\mathrm{p}=0.459$, respectively). There was no significant change in the ejection fraction at either follow-up (p>0.05 for all) (Fig. 3).

Although RVEDVi and RVESVi improved after ASD closure, the RV volume parameters were still larger than the LV volume parameters at the 1-year follow-up (RVEDVi versus LVEDVi: $\mathrm{p}=0.016$; RVESVi versus LVESVi: $\mathrm{p}=0.001$ ). In addition, the RVEF was lower than the LVEF at the 1 -year postoperative follow-up $(\mathrm{p}<0.001)$ (Table 2$)$.

\section{Late gadolinium enhancement}

LGE was detected in 12 patients (92.3\%) on preoperative $\mathrm{CMR}$ at the RV basal septum insertion point. This LGE resolved in 1 patient $(7.7 \%)$, was maintained with a slight decrease in 4 patients (30.8\%), and was maintained in 7 patients (53.8\%) at the 1-year postoperative follow-up (Fig. 4).

\section{Discussion}

In this study, patients' RV volume had considerably decreased at the 1-month postoperative follow-up and showed a steady decrease until the 1-year follow-up. Thus, RV adaptation occurred rapidly and gradual RV remodeling continued until 1 year postoperatively. This ventricular adaptation is similar to the results reported in other studies on ASD patients [4-7]. The cause of early rapid RV adaptation
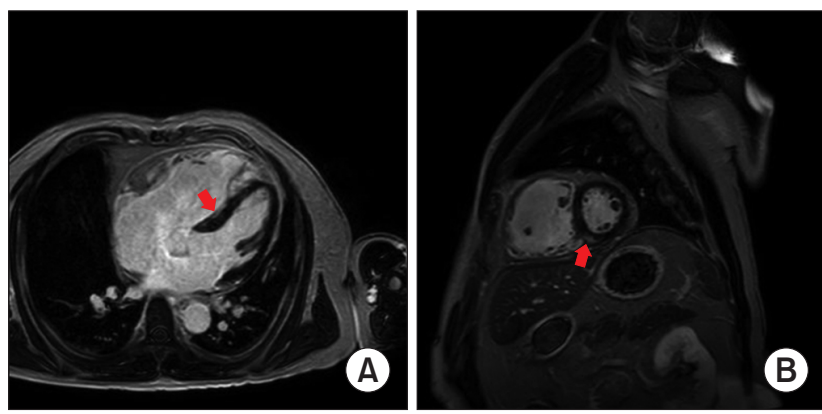

Fig. 4. (A, B) Late gadolinium enhancement at the right ventricle basal septum insertion point on cardiac magnetic resonance imaging. The arrow indicates fibrosis.

may have been the disappearance of the left-to-right shunt. However, we found that the RV volume was larger than the $\mathrm{LV}$ volume at the 1-year postoperative follow-up, despite the statistically significant decrease in RV volume. In this study, patients' median age of 51.5 years combined with their median Qp/Qs of 2.3 may have been responsible for long-term RV volume overload, because Qp/Qs and RVEDVi showed a positive correlation. A relatively long period of $\mathrm{RV}$ volume overload may affect $\mathrm{RV}$ remodeling. In another report, $20 \%$ of children with a median age of 7.5 years had a larger RV volume than LV volume after surgical ASD closure. Thus, ASD closure in early life does not guarantee a complete normalization of the heart chamber size [8].

In this study, we found that the RVEF had been maintained in a nearly below-normal state at the 1-year postoperative follow-up. Some reports have explained persistent $\mathrm{RV}$ dysfunction for up to several years by the trauma of operation, the use of a cardioplegic solution, and adverse inflammatory responses [9-13]. These exacerbating factors may have contributed to the persistent RV dysfunction observed in follow-up in this study. However, we found that 
LGE at the RV basal septum insertion point was detected in $92.3 \%$ of patients in this study, but only completely resolved in one patient. The other patients had partial resolution or no change. The sources of persistent LGE may induce RV strain and cause RV dysfunction in adult patients.

The patients who had palpitation during the preoperative period did not show symptom improvement during follow-up in this study. Several reports have stated that atrial remodeling is less effective than ventricular remodeling and is associated with atrial arrhythmia. The right atrial stretch in ASD may cause electrophysiological alterations, and these permanent structural atrial abnormalities may be a cause of atrial arrhythmia in affected patients $[14,15]$. Although we unfortunately did not measure atrial changes in this study, the lack of improvement in the patients' arrhythmic symptoms may be related to atrial remodeling.

In this study, the preoperative median amount of TVR was $14.1 \%$. We only performed tricuspid valve repair in 1 patient who had tricuspid valve annular dilatation with more than a moderate degree of TVR. The post-ASD closure TVR was resolved at the 1-year postoperative follow-up. In 1 report, the resolution of TVR was described as a result of RV restoration and not of structural changes in the tricuspid valve annulus [6]. Although we did not measure the tricuspid valve annulus diameter on CMR, we suspect that RV volume resolution may affect TVR resolution.

There are several limitations of this study. First, we did not perform exercise cardiopulmonary function testing. Thus, we did not find any correlation between RV volume and the results of exercise tests. Second, the sample size was small because of the cost of CMR, resulting in limited statistical power.

In conclusion, RV adaptation after ASD closure occurred rapidly and gradual RV remodeling continued for 1 year. However, RV remodeling did not reach complete normalization. In addition, LGE was detected and showed partial resolution or no change after ASD closure in almost all patients.

\section{Conflict of interest}

No potential conflict of interest relevant to this article was reported.

\section{Funding}

This work was supported by a National Research Foundation of Korea (NRF) grant funded by the Korean gov- ernment (No. 2017R1C1B5076818).

\section{ORCID}

Yo Han Bae: https://orcid.org/0000-0001-7054-1898

Woo Sung Jang: https://orcid.org/0000-0003-1576-9472

Jin Young Kim: https://orcid.org/0000-0001-6714-8358

Yun Seok Kim: https://orcid.org/0000-0002-2817-557X

\section{References}

1. Hoffman JI, Kaplan S, Liberthson RR. Prevalence of congenital heart disease. Am Heart J 2004;147:425-39.

2. Baumgartner H, Bonhoeffer P, De Groot NM, et al. ESC guidelines for the management of grown-up congenital heart disease (new version 2010). Eur Heart J 2010;31:2915-57.

3. Kutty S, Hazeem AA, Brown K, et al. Long-term (5- to 20-year) outcomes after transcatheter or surgical treatment of hemodynamically significant isolated secundum atrial septal defect. Am J Cardiol 2012; 109:1348-52

4. Thilen U, Persson S. Closure of atrial septal defect in the adult: cardiac remodeling is an early event. Int J Cardiol 2006;108:370-5.

5. Teo KS, Dundon BK, Molaee P, et al. Percutaneous closure of atrial septal defects leads to normalisation of atrial and ventricular volumes. J Cardiovasc Magn Reson 2008;10:55.

6. Schoen SP, Kittner T, Bohl S, et al. Transcatheter closure of atrial septal defects improves right ventricular volume, mass, function, pulmonary pressure, and functional class: a magnetic resonance imaging study. Heart 2006;92:821-6.

7. Pascotto M, Santoro G, Cerrato F, et al. Time-course of cardiac remodeling following transcatheter closure of atrial septal defect. Int J Cardiol 2006;112:348-52.

8. Roos-Hesselink JW, Meijboom FJ, Spitaels SE, et al. Excellent survival and low incidence of arrhythmias, stroke and heart failure longterm after surgical ASD closure at young age: a prospective follow-up study of 21-33 years. Eur Heart J 2003;24:190-7.

9. Ascione R, Lloyd CT, Underwood MJ, Lotto AA, Pitsis AA, Angelini GD. Inflammatory response after coronary revascularization with or without cardiopulmonary bypass. Ann Thorac Surg 2000;69:1198204.

10. Tarnok A, Hambsch J, Emmrich F, et al. Complement activation, cytokines, and adhesion molecules in children undergoing cardiac surgery with or without cardiopulmonary bypass. Pediatr Cardiol 1999; 20:113-25.

11. Zahler S, Massoudy P, Hartl H, Hahnel C, Meisner H, Becker BF. Acute cardiac inflammatory responses to postischemic reperfusion during cardiopulmonary bypass. Cardiovasc Res 1999;41:722-30.

12. Breisblatt WM, Stein KL, Wolfe CJ, et al. Acute myocardial dysfunction and recovery: a common occurrence after coronary bypass 
surgery. J Am Coll Cardiol 1990;15:1261-9.

13. Chaturvedi RR, Lincoln C, Gothard JW, et al. Left ventricular dysfunction after open repair of simple congenital heart defects in infants and children: quantitation with the use of a conductance catheter immediately after bypass. J Thorac Cardiovasc Surg 1998;115:7783.
14. Kort HW, Balzer DT, Johnson MC. Resolution of right heart enlargement after closure of secundum atrial septal defect with transcatheter technique. J Am Coll Cardiol 2001;38:1528-32.

15. Morton JB, Sanders P, Vohra JK, et al. Effect of chronic right atrial stretch on atrial electrical remodeling in patients with an atrial septal defect. Circulation 2003;107:1775-82. 